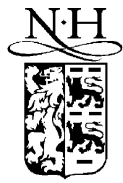

ELSEVIER

\title{
PP-waves from BPS supergravity monopoles
}

\author{
D.H. Correa ${ }^{\mathrm{a}, 1}$, E.F. Moreno ${ }^{\mathrm{a}, 2}$, S. Reuillon ${ }^{\text {b,a }}$, F.A. Schaposnik ${ }^{\mathrm{a}, 3}$ \\ a Departamento de Física, Universidad Nacional de La Plata, C.C. 67, 1900 La Plata, Argentina \\ ${ }^{\mathrm{b}}$ Laboratoire de Mathématiques et Physique Théorique, CNRS/UMR 6083, Université de Tours, Parc de Grandmont, 37200 Tours, France
}

Received 12 November 2002; accepted 3 December 2002

Editor: L. Alvarez-Gaumé

\begin{abstract}
We discuss the Penrose limit of the Chamseddine-Volkov BPS selfgravitating monopole in four-dimensional $N=4$ supergravity theory with non-Abelian gauge multiplets. We analyze the properties of the resulting supersymmetric pp-wave solutions when various Penrose limits are considered. Apart from the usual rescaling of coordinates and fields we find that a rescaling of the gauge coupling constant to zero is required, rendering the theory Abelian. We also study the Killing spinor equations showing an enhancement of the supersymmetries preserved by the solutions and discuss the embedding of the pp-wave solution in $d=10$ dimensions.
\end{abstract}

๑) 2002 Elsevier Science B.V. All rights reserved.

Plane wave backgrounds arising in supergravity theories have recently attracted much attention in connection with the solution of superstring theory and its relation with large $N$ gauge theory [1-9]. Such parallel plane (pp) wave solutions can be obtained by taking the so-called Penrose limit [10] adapted to the case of supergravity theories [11]. In the present Letter we follow this approach to find pp-wave solutions in $N=4$ gauged supergravity in 4 dimensions [12], by starting from the BPS selfgravitating monopole solution constructed by Chamseddine and Volkov some time ago $[13,14]$. In taking the Penrose limit, we had to adapt Güven treatment [11] to the case of

\footnotetext{
E-mail address: emoreno@fisica.unlp.edu.ar (E.F. Moreno).

1 CONICET.

2 Associated with CONICET.

3 Associated with CICPBA.
}

gauged supergravity finding that not only fields but also the gauge coupling constant should be scaled in order to find consistent pp-wave solutions. In fact, the coupling constant is rescaled to zero and the ppwave solution corresponds to an Abelianized version of the theory. We consider different limits and discuss their relation as a consequence of the covariance property of the Penrose limit as originally discussed in [6]. We also analize the uplifting of the solution to 10 -dimensional $N=1$ supergravity and discuss the issue of preserved supersymmetries.

We start by reviewing the $d=4$ gauged supergravity model and its BPS monopole solution and then discuss the Penrose limit and the resulting pp-wave configurations. Afterwards, we discuss how a $d=10$ uplifted solution can be obtained from the $d=4$ theory by an appropriate choice of the gauge group manifold. 
The starting point is a four-dimensional $S U(2) \times$ $S U$ (2) $N=4$ supergravity theory with non-Abelian Yang-Mills multiplets [12]. The field content of the theory includes, in the bosonic sector, vierbein fields $e_{\mu}^{a}$, vector and pseudo-vector non-Abelian gauge fields $A_{\mu}^{a}$ and $B_{\mu}^{a}(a=1,2,3)$, a dilaton $\phi$, and an axion a. Concerning the fermionic sector, there are 4 Majorana spin-3/2 fields $\psi_{\mu}^{I}$ and 4 Majorana spin-1/2 fields $\chi^{I}$ $(I=1,2,3,4)$.

In the Chamseddine-Volkov (CV) monopole solution $[13,14]$ the pseudovector gauge field $B_{\mu}^{a}$ and the axion $\mathbf{a}$ are put to zero. The bosonic part of the action then reduces to

$$
\begin{aligned}
S=\int & \sqrt{-g} d^{4} x \\
\times & \left(-\frac{1}{4} R+\frac{1}{2} \partial_{\mu} \phi \partial^{\mu} \phi-\frac{1}{4} \exp (2 \phi) F_{\mu \nu}^{a} F^{a \mu \nu}\right. \\
& \left.+\frac{e^{2}}{8} \exp (-2 \phi)\right),
\end{aligned}
$$

where

$$
F_{\mu \nu}^{a}=\partial_{\mu} A_{\nu}^{a}-\partial_{\nu} A_{\mu}^{a}+e \epsilon^{a b c} A_{\mu}^{b} A_{\nu}^{c} .
$$

For purely bosonic configurations the supersymmetry transformation laws reduce to

$$
\begin{aligned}
\delta \bar{\chi}= & -\frac{i}{\sqrt{2}} \bar{\epsilon} \gamma^{\mu} \partial_{\mu} \phi-\frac{1}{2} \exp (\phi) \bar{\epsilon} F_{\mu \nu} \sigma^{\mu \nu} \\
& +\frac{e}{4} \exp (-\phi) \bar{\epsilon}, \\
\delta \bar{\psi}_{\rho}= & \nabla_{\rho} \bar{\epsilon}+\bar{\epsilon} \frac{e}{2} A_{\rho}-\frac{i}{2 \sqrt{2}} \exp (\phi) \bar{\epsilon} F_{\mu \nu} \gamma_{\rho} \sigma^{\mu \nu} \\
& +\frac{i e}{4 \sqrt{2}} \exp (-\phi) \bar{\epsilon} \gamma_{\rho} .
\end{aligned}
$$

Here we have written $A_{\mu}=A_{\mu}^{a} \alpha^{a}$, where $\alpha_{I J}^{a}$ are three of the six $4 \times 4$ matrices which generate the algebra of the original $S U(2) \times S U(2)$ symmetry group (an explicit representation constructed in terms of the Pauli matrices is given in [12]). We have also used $\sigma^{\mu \nu}=\frac{1}{4}\left[\gamma^{\mu}, \gamma^{\nu}\right]$ and we have written the 4 Majorana spinor parameters as $\epsilon^{I} \equiv \epsilon . \nabla_{\rho} \bar{\epsilon}$ stands for the spinorial covariant derivative

$\nabla_{\rho} \bar{\epsilon}=\partial_{\rho} \bar{\epsilon}-\frac{1}{2} \bar{\epsilon} \omega_{\rho}^{\alpha \beta} \sigma_{\alpha \beta}$,

where $\omega_{\rho}^{\alpha \beta}$ is the spin connection (earlier Greek letters $\alpha, \beta, \ldots$, correspond to the locally flat system).
The spherically symmetric CV exact solution was found by integrating the BPS equations that result from the vanishing of the supersymmetry transformations (3), (4). The explicit form of the metric and dilaton is given by

$$
\begin{aligned}
& d s^{2}=2 \exp (2 \phi)\left(d t^{2}-\frac{1}{e^{2}} d \rho^{2}\right. \\
& \left.\quad-\frac{R^{2}(\rho)}{e^{2}}\left(d \theta^{2}+\sin ^{2} \theta d \varphi^{2}\right)\right), \\
& \exp (2 \phi)=a^{2} \frac{\sinh \rho}{2 R(\rho)}, \\
& R^{2}(\rho)=2 \rho \operatorname{coth} \rho-\frac{\rho^{2}}{\sinh ^{2} \rho}-1,
\end{aligned}
$$

where $a$ is a free parameter reflecting scaling invariance of the equations of motion. Concerning the gauge field, the solution takes the form

$$
\begin{aligned}
& \alpha^{a} A_{\mu}^{a} d x^{\mu} \\
& \quad=\frac{w}{e}\left(-\alpha^{2} d \theta+\alpha^{1} \sin \theta d \varphi\right)+\frac{1}{e} \alpha^{3} \cos \theta d \varphi, \\
& w= \pm \frac{\rho}{\sinh \rho} .
\end{aligned}
$$

Here $\rho$ is a radial-like variable $(0 \leqslant \rho<\infty)$ implicitly defined in terms of $w$ and $\phi$ while $a$ is a free parameter which reflects the scale symmetry of Bogomol'nyi equations. The geometry described by the metric (6) is everywhere regular and corresponds to a space whose topology is $\mathbb{R}^{4}$. Concerning the gauge field solution, where $\rho$ the standard radial variable, it would exactly coincide with the well-honored flat-space BPS gauge field solution $[15,16]$, which corresponds to a charge 1 magnetic monopole. In the present case, being all gauge field massless, the asymptotic behavior of $w$ in terms of the physical radial variable $r$ is not exponentially decaying but

$w \sim \frac{\log r}{r^{2}}$ for $r \rightarrow \infty$.

As already noted in $[13,14]$, defining a magnetic charge for this gauge field configuration is problematic since there is no Higgs field breaking the symmetry and providing a natural isospin direction to project the $S U$ (2) field strength on the direction of the residual Abelian symmetry (as one does for the original flat space 't Hooft-Polyakov (tHP) monopole configuration). Note however that ansatz (9) is nothing but 
the gauge-transformed (with element $S$ ) of the original tHP ansatz (we call $\bar{A}$ the gauge field ansatz in its original tHP form) entangling space and isospace indices,

$A \rightarrow \bar{A}=S^{-1} A S+\frac{i}{e} S^{-1} d S=\frac{i}{e}(1-w)[\Omega, d \Omega]$,

$\Omega=\frac{\alpha^{a}}{2} \frac{x^{a}}{r}$.

Then, as for the pure Yang-Mills pioneering monopole ansatz of Wu and Yang [17], one can define a projected field strength in the form

$\overline{\mathcal{F}}_{\mu \nu}=\operatorname{Tr}\left(\bar{F}_{\mu \nu} \Omega\right)$

which, in the $\mathrm{CV}$ gauge becomes

$\mathcal{F}_{\mu \nu}=\operatorname{Tr}\left(F_{\mu \nu} S \Omega S^{-1}\right)$

leading to a magnetic field $\mathcal{B}_{i}=(1 / 2) \varepsilon_{i j k} \mathcal{F}_{i j}$ which takes the form

$\mathcal{B}_{r}=\left(1-w^{2}\right)$

which indeed corresponds to a charge 1 magnetic monopole.

The Penrose limit procedure expands outwards the immediate neighborhood of a null geodesic. Then, one has several possibilities both in choosing the particular pair of variables entering in the definition of the "light-cone" variables and how one redefines and shifts the remaining variables. We shall explore two, in principle, different Penrose limits of the solution described above, and also discuss their relation.

The first Penrose limit is taken along a radial $\theta=\pi / 2$ (and $\varphi=0$ ) null geodesic. The appropriate change of coordinates is in this case

$t=\frac{1}{2}\left(u-\Omega^{2} v\right), \quad \rho=\frac{e}{2}\left(u+\Omega^{2} v\right)$,

$\theta=\Omega y+\frac{\pi}{2}, \quad \varphi=\Omega x$,

where $\Omega$ is a positive real parameter.

The next stage corresponds to redefine the fields through an appropriate scaling so that every term in the Lagrangian is scaled by the same factor [11]. The fields redefinitions are the following

$\bar{g}_{\mu \nu}=\Omega^{-2} g_{\mu \nu}$,

$\bar{A}=\Omega^{-1} A$.
Moreover, to obtain a homogenous power of $\Omega$ in front of the Lagrangian, we should scale the gauge coupling constant

$\bar{e}=\Omega e$.

Let us perform this scaling on solution (6)-(9). Now, the expressions of the new fields are

$$
\begin{aligned}
& d \bar{s}^{2}=-2 \exp (2 \phi) \\
& \times\left(d u d v+\frac{R^{2}}{e^{2}}\left(d y^{2}+\cos ^{2}(\Omega y) d x^{2}\right)\right), \\
& \exp (2 \phi)=a^{2} \frac{\sinh \left(\frac{e}{2}\left(u+\Omega^{2} v\right)\right)}{2 R\left(\frac{e}{2}\left(u+\Omega^{2} v\right)\right)} \\
& \alpha^{a} \bar{A}_{\mu}^{a} d x^{\mu}=\frac{w}{e}\left(-\alpha^{2} d y+\alpha^{1} \cos (\Omega y) d x\right) \\
&-\frac{1}{e} \alpha^{3} \sin (\Omega y) d x .
\end{aligned}
$$

Then, the Penrose limit is accomplished by taking $\Omega \rightarrow 0$. It is worth noting that in this limit, the scaled coupling constant (17) goes to zero and, as consequence, there is no commutator in the field strengths. One can then consider the field configuration obtained after the Penrose limit as a solution of an Abelianized version of the theory (1). In this sense the Penrose limit relates solutions of a gauge supergravity theory to solutions of a different gauge supergravity theory. The explicit form of the solution is

$$
\begin{aligned}
& d \bar{s}^{2}=-2 \exp (2 \phi)\left(d u d v+\frac{R^{2}}{e^{2}}\left(d y^{2}+d x^{2}\right)\right), \\
& \exp (2 \phi)=a^{2} \frac{\sinh \left(\frac{e}{2} u\right)}{2 R\left(\frac{e}{2} u\right)}, \\
& \alpha^{a} \bar{A}_{\mu}^{a} d x^{\mu}=\frac{w}{e}\left(\alpha^{1} d x-\alpha^{2} d y\right) .
\end{aligned}
$$

It is straightforward to check that this pp-wave configuration, fulfills the equations of motion. Moreover, as it is the case for the original CV solution, it is everywhere regular. Concerning the pp-wave metric, it can be written in Rosen form if one redefines the $u$ variable so that the dilaton factor is eliminated from $g_{u v}$. Concerning the gauge field solution, one can associate $A_{\mu}^{1}$ and $A_{\mu}^{2}$ with two $U(1)$ gauge fields with electric and magnetic fields given by

$$
F_{t x}^{1}=\frac{1}{e} \partial_{t} w\left(\frac{\rho+e t}{2}\right),
$$


$F_{\rho x}^{1}=\frac{1}{e} \partial_{\rho} w\left(\frac{\rho+e t}{2}\right)$,

so that $F_{t x}^{1}=e F_{\rho x}^{1}$, thus corresponding to a plane wave travelling along the $\rho$ direction with orthogonal electric and magnetic fields (for the other $U(1)$ gauge field one gets an analogous result, with $y$ coordinate in place of $x$ ). Notice that $F_{\mu \nu}^{i} F^{i \mu \nu}=0$ and ${ }^{*} F_{\mu \nu}^{i} F^{i \mu \nu}=0(i=1,2)$, that is, the solution corresponds to a null field of the kind arising for other plane-wave Einstein-Maxwell solutions. Let us comment that if one uses the monopole gauge field configuration as in the original 't Hooft-Polyakov ansatz instead of its gauge transformed version (11), one arrives to the same pp-wave solution (with the nontrivial gauge field components in a different isospin direction).

As we stated above, we shall analyze a different Penrose limit taken along a radial $\theta=0$ null geodesic. Of course, this geodesic belongs to the same orbit under the isometry group as that corresponding to $\theta=\pi / 2$. Then, as a consequence of the covariance property of the Penrose limit introduced in [6], the solutions for the metrics should coincide. Now, in the present case, it is interesting to analyse how the corresponding gauge field solutions are connected. In fact, we shall see that field configurations are related by a (singular) gauge transformation.

The change of variables reads in this case

$t=\frac{1}{2}\left(u-\Omega^{2} v\right), \quad \rho=\frac{e}{2}\left(u+\Omega^{2} v\right)$,

$\theta=\Omega r, \quad \varphi=\varphi$.

The metric, gauge field and coupling constant scaling is the same as in the previous case (Eqs. (16), (17)). Proceeding exactly as before we arrive, after taking the $\Omega \rightarrow 0$ limit, to the following pp-wave solution,

$$
\begin{aligned}
& d \bar{s}^{2}=-2 \exp (2 \phi) \\
& \times\left(d u d v+\frac{R^{2}}{e^{2}}\left(d r^{2}+r^{2} d \varphi^{2}\right)\right), \\
& \exp (2 \phi)=a^{2} \frac{\sinh \left(\frac{e}{2} u\right)}{2 R\left(\frac{e}{2} u\right)}, \\
& \bar{A}_{\varphi}^{1}=\frac{w}{e} r, \quad \bar{A}_{r}^{2}=-\frac{w}{e} .
\end{aligned}
$$

Concerning the $\bar{A}_{\varphi}^{3}$ component, before taking the $\Omega \rightarrow 0$ limit one has

$\bar{A}_{\varphi}^{3}=\frac{1}{e \Omega} \cos (\Omega r)$.

The corresponding nontrivial field strengths components are

$$
\begin{aligned}
& \bar{F}_{t \varphi}^{1}=\frac{1}{e} r \partial_{t} w\left(\frac{\rho+e t}{2}\right), \\
& \bar{F}_{\rho \varphi}^{1}=\frac{1}{e} r \partial_{\rho} w\left(\frac{\rho+e t}{2}\right), \\
& \bar{F}_{t r}^{2}=-\frac{1}{e} \partial_{t} w\left(\frac{\rho+e t}{2}\right), \\
& \bar{F}_{\rho r}^{2}=-\frac{1}{e} \partial_{\rho} w\left(\frac{\rho+e t}{2}\right) .
\end{aligned}
$$

Note that the $\Omega \rightarrow 0$ divergence in $\bar{A}_{\varphi}^{3}$ is not harmful since this component has an associated field strength $\bar{F}^{3}=0$. Concerning its contribution to $\bar{F}^{1}$ and $\bar{F}^{2}$, the factor $\bar{e}=\Omega e$ in front of commutators cancels out the singularity. Moreover, to order $1 / \Omega$ the $\bar{A}_{\varphi}^{3}$ component can be gauged out by performing a (singular) gauge transformation

$g=\exp \left(\alpha_{3} \frac{\varphi}{2}\right)$

The gauge transformed field strength components read,

$$
\begin{array}{rlrl}
{ }^{g} \bar{F}_{t \varphi}^{1} & =\frac{1}{e} r \cos \varphi \partial_{t} w, & { }^{g} \bar{F}_{\rho \varphi}^{1}=\frac{1}{e} r \cos \varphi \partial_{\rho} w, \\
{ }^{g} \bar{F}_{t r}^{1}=-\frac{1}{e} \sin \varphi \partial_{t} w, & { }^{g} \bar{F}_{\rho r}^{1}=-\frac{1}{e} \sin \varphi \partial_{\rho} w
\end{array}
$$

and analogous expressions for the ${ }^{g} \bar{F}^{2}$ components. As advanced these expressions for ${ }^{g} \bar{F}^{1}$ (and the corresponding one for ${ }^{g} \bar{F}^{2}$ ), obtained by choosing the Penrose limit according to Eq. (23) coincide with those resulting from the alternative Penrose limit (15). Indeed, Eqs. (22) and (30) coincide if one writes in Cartesian coordinates the latter.

It is interesting to note that the field strength, written in the form (28), can be interpreted in terms of a (gauge-dependent) magnetic field. Indeed, the $\bar{F}_{\rho \varphi}^{1}$ component can be associated to a radial magnetic field 
with flux

$$
\begin{aligned}
\Phi & =\frac{1}{2} \int \varepsilon_{i j k} \bar{F}_{j k}^{1} d S_{i} \\
& =\frac{1}{e} \int_{0}^{2 \pi} d \varphi \int_{-e t}^{\infty} d \rho \partial_{\rho} w\left(\frac{\rho+e t}{2}\right)=\frac{4 \pi}{e}
\end{aligned}
$$

which corresponds to a unit magnetic charge, as for the original CV monopole solution (see Eq. (14)). Of course, as in this last case, one is dealing with gauge dependent fluxes.

The analysis above on two isometric null geodesics leading to limiting solutions that should be identified, shows the relevance of classifying those Penrose limits that, in contrast, are inequivalent. This implies determining orbits of the isometry group of space time on the space of null geodesics. This has been done for spaces of the form $A d S \times S$ and supergravity brane solutions in [6]. For classifying the Penrose limit in the case of the CV monopole solution, one should start by observing that the resulting family of non-isometric Penrose limits can be labelled by the angular momentum $l$ of the corresponding null geodesic. We have already presented above the pp-waves configurations arising by taking the limit along radial null geodesics $(l=0)$. The classification should then be completed by studying the Penrose limit along a generic null geodesic. The geodesic equations are in this case more involved and deserve a careful analysis that we shall present elsewhere.

It is known that the Penrose limit can enhance the number of preserved supersymmetries. We will see that this is the case for the solution we are analyzing. Indeed, the original CV solution preserves $1 / 4$ of the supersymmetries while, as we shall see, the pp-wave solution Eqs. (19)-(21) preserves 1/2. To see this, let us find the Killing spinors $\epsilon$ making supersymmetry variations (3), (4) vanish. The corresponding equations read

$$
\begin{aligned}
\delta \bar{\chi}= & \frac{\exp (-\phi)}{\sqrt{2}} \bar{\epsilon} \gamma^{\underline{u}} \\
& \times\left(-i \partial_{u} \phi-\left(\alpha^{1} \gamma^{\underline{x}}-\alpha^{2} \gamma^{\underline{y}}\right) \frac{\partial_{u} w}{2 R(u)}\right)=0,
\end{aligned}
$$

$$
\begin{aligned}
\delta \bar{\psi}_{\mu}= & \nabla_{\mu} \bar{\epsilon}-i \bar{\epsilon} \gamma_{\mu} \gamma^{\underline{u}}\left(\alpha^{1} \gamma^{\underline{x}}-\alpha^{2} \gamma^{\underline{y}}\right) \\
& \times \exp (-\phi) \frac{\partial_{u} w}{4 R(u)}=0,
\end{aligned}
$$

where underlined indices in gamma matrices refer to the tangent space. Now, any spinor $\bar{\chi}$ can be written as

$\bar{\chi}=\bar{\chi}^{1} \gamma^{\underline{v}}+\bar{\chi}^{2} \gamma^{\underline{u}}$

where

$\chi^{1}=-\frac{1}{2} \gamma_{\underline{u}} \chi, \quad \chi^{2}=-\frac{1}{2} \gamma_{\underline{v}} \chi$,

so that if we make the choice

$\bar{\epsilon}(u, v, x, y)=\bar{\epsilon}^{1}(u, v, x, y) \gamma^{\underline{u}}$,

Eq. (32) is automatically satisfied. Concerning (33), since $\omega_{\mu}^{\alpha \beta} \sigma_{\alpha \beta} \gamma^{u}=0$, for $\mu=v, x, y$ it reduces to

$\partial_{\mu} \bar{\epsilon}^{1}(u, v, x, y) \gamma^{\underline{u}}=0$

so that $\epsilon^{1}=\epsilon^{1}(u)$, whereas the remaining equation reads

$$
\left(\partial_{u} \bar{\epsilon}^{1}(u)+i \bar{\epsilon}^{1}(u)\left(\alpha^{1} \gamma^{\underline{x}}-\alpha^{2} \gamma^{\underline{y}}\right) \frac{\partial_{u} w}{2 R(u)}\right) \gamma^{\underline{u}}=0
$$

which can be easily integrated. We then conclude that at least the pp-wave solution preserves $1 / 2$ of the 16 supersymmetries of the theory. To determine whether there are additional Killing spinors, one should look for spinors of the form $\bar{\epsilon}^{2}(u, v, x, y) \gamma^{-} \underline{v}$ with vanishing supersymmetry variation. But then, from Eq. (32) one concludes that the following equation should hold,

$\bar{\epsilon}^{2} \gamma^{\underline{v}} \gamma^{\underline{u}}\left(-i \partial_{u} \phi-\left(\alpha^{1} \gamma^{\underline{x}}-\alpha^{2} \gamma^{\underline{y}}\right) \frac{\partial_{u} w}{2 R(u)}\right)=0$.

Now, in view of the explicit $u$-dependence of $\phi, R$ and $w$ given in (7)-(9), the only possibility is that $\epsilon^{2}=0$. In conclusion, one only finds the standard Killing spinors and hence the supersymmetries preserved by the pp-wave are $1 / 2$, that is, twice those preserved by $\mathrm{CV}$ solution.

Four-dimensional solutions like the original monopole solution (6)-(9) or the pp-wave solution (19)(21) can be uplifted to $d=10$ dimensions as solutions of $N=1$ supergravity. Concerning the former, this was done in [14] and the same procedure can be used to uplift the latter. We shall here briefly describe the last uplifting. 
Following [14], we shall use a hat to distinguish fields in ten dimensions and Latin capital letters refer to the coordinates. The sets $(M, N, P, \ldots)$ and $(A, B, C, \ldots)$ stand for curved space and tangent space indices, respectively. These sets can be decomposed into space-time and internal indices from the 4-dimensional viewpoint. That is, $\{M\}=\{\mu, m\}$ and $\{A\}=\{\alpha, \mathrm{a}\}(\mu, \alpha=0,1,2,3$ and $m, \mathrm{a}=4,5, \ldots, 9)$.

The action for the $N=1$ supergravity in $d=10$ is

$$
\begin{aligned}
\widehat{S}=\int & \sqrt{-\hat{g}} d^{10} x \\
\times & \left(-\frac{1}{4} \widehat{R}+\frac{1}{2} \partial_{\mu} \hat{\phi} \partial^{\mu} \hat{\phi}\right. \\
& \left.\quad+\frac{1}{12} \exp (-2 \hat{\phi}) \widehat{H}_{M N P} \widehat{H}^{M N P}\right),
\end{aligned}
$$

where the antisymmetric field strength tensor is given in terms of a two-form gauge field

$$
\widehat{H}_{M N P}=\partial_{M} \widehat{B}_{N P}+\partial_{N} \widehat{B}_{P M}+\partial_{P} \widehat{B}_{M N} .
$$

The reduction from $d=10$ to $d=4$ can be done in many different ways. Depending on the sixdimensional manifold $\mathcal{M}_{6}$ used to compactify the original $d=10$ space to $d=4$ space one obtains different $d=4$ gauged supergravity. For instance, compactifying with $S^{3} \times S^{3}$ or $S^{3} \times T^{3}$ the resulting supergravities in $d=4$ are gauged by $S U(2) \times S U(2)$ or $S U(2) \times[U(1)]^{3}$, respectively.

If the manifold spanned by $\left\{x^{m}\right\}$ forms a compact group space, there will be functions satisfying

$$
\left(U^{-1}\right)_{\mathrm{b}}{ }^{m}\left(U^{-1}\right)_{\mathrm{c}}{ }^{n}\left(\partial_{m} U^{\mathrm{a}}{ }_{n}-\partial_{n} U^{\mathrm{a}}{ }_{m}\right)=\frac{f_{\mathrm{abc}}}{\sqrt{2}} .
$$

Let us present the ansatz for expressing the 10-dimensional fields in terms of their 4-dimensional counterparts and the functions $U^{\mathrm{a}}{ }_{m}$. The 10-dimensional metric tensor is

$$
\begin{aligned}
& \hat{g}_{\mu \nu}=\exp (-3 \phi / 2) g_{\mu \nu}-2 \exp (\phi / 2) A^{\mathrm{a}}{ }_{\mu} A^{\mathrm{a}}{ }_{\nu}, \\
& \hat{g}_{\mu m}=\sqrt{2} \exp (\phi / 2) A^{\mathrm{a}}{ }_{\mu} U^{\mathrm{a}}{ }_{m}, \\
& \hat{g}_{m n}=-\exp (\phi / 2) U^{\mathrm{a}}{ }_{m} U^{\mathrm{a}}{ }_{n} .
\end{aligned}
$$

The ansatz for the dilaton is simply

$$
\hat{\phi}=-\frac{\phi}{2} \text {. }
$$

Finally, the 10-dimensional field strength tensor takes the form

$$
\begin{aligned}
\widehat{H}_{\alpha \beta \gamma} & =\exp (-7 \phi / 4) \varepsilon_{\delta \alpha \beta \gamma} \partial^{\delta} \mathbf{a}, \\
\widehat{H}_{\alpha \beta \mathrm{a}} & =-\frac{1}{\sqrt{2}} \exp (5 \phi / 4) F_{\alpha \beta}^{\mathrm{a}}, \\
\widehat{H}_{\alpha \mathrm{ab}} & =0, \\
\widehat{H}_{\mathrm{abc}} & =\frac{1}{2 \sqrt{2}} \exp (-3 \phi / 4) f_{\mathrm{abc}} .
\end{aligned}
$$

Some comments are in order. The field a, appearing in Eq. (45) is the axion, which is set to zero in the solutions that we will uplift. The field strength in (46) takes values in the Lie algebra of the six-dimensional manifold

$$
F_{\alpha \beta}^{\mathrm{a}}=\partial_{\alpha} A^{\mathrm{a}}{ }_{\beta}-\partial_{\beta} A^{\mathrm{a}}{ }_{\alpha}+f_{\mathrm{abc}} A^{\mathrm{b}}{ }_{\alpha} A^{\mathrm{c}}{ }_{\beta} .
$$

We shall now proceed to construct a pp-wave solution to the equations of motion of the $d=10$ supergravity model. As stated above, one can directly uplift to this end, the $d=4$ pp-wave solution (19)(21).

The pp-wave solution (19)-(21) involves just two gauge field components $A_{\mu}^{1}$ and $A_{\mu}^{2}$ which, being the gauge coupling constant zero in the Penrose limit, lead to two Abelian field strengths. It is then natural to choose the six-dimensional group manifold so as to arrive to a model with just Abelian gauge fields, namely $\mathcal{M}_{6}=T^{6}$. The appropriate choice of functions $U^{\mathrm{a}}{ }_{m}$ is

$U^{\mathrm{a}}{ }_{m}=\delta^{\mathrm{a}}{ }_{m}$.

This choice, together with the explicit form for $A_{\mu}^{1}$ and $A_{\mu}^{2}$ given in (19)-(21), leads to the following tendimensional pp-wave metric,

$$
\begin{aligned}
\hat{g}_{\mu \nu}= & \exp (-3 \phi / 2) g_{\mu \nu} \\
& -2 \exp (\phi / 2) \frac{w^{2}}{e^{2}}\left(\delta_{\mu}^{x} \delta_{v}^{x}+\delta_{\mu}^{y} \delta_{v}^{y}\right), \\
\hat{g}_{x 4}= & \sqrt{2} \exp (\phi / 2) \frac{w}{e}, \\
\hat{g}_{y 5}= & -\sqrt{2} \exp (\phi / 2) \frac{w}{e}, \\
\hat{g}_{m n}= & -\exp (\phi / 2) \delta_{m n} .
\end{aligned}
$$

Concerning the field strength components, the only non-vanishing ones take the form

$$
\widehat{H}_{u x 4}=-\frac{1}{\sqrt{2} e} \frac{d w}{d u},
$$


$\widehat{H}_{u y 5}=\frac{1}{\sqrt{2} e} \frac{d w}{d u}$.

Finally, the solution for the dilaton is given by

$\exp (4 \hat{\phi})=\exp (2 \phi)=a^{2} \frac{\sinh \left(\frac{e}{2} u\right)}{2 R\left(\frac{e}{2} u\right)}$.

We have checked, after a straightforward but tedious computation, that the pp-wave configuration (51)(53) is a solution of the $d=10, N=1$ supergravity equations of motion.

In summary, we have studied the Penrose limit of the Chamseddine-Volkov BPS monopole solution to the equations of motion of $N=4$ supergravity theory with non-Abelian gauge multiplets in $d=4$ dimensions. We found that in order to define a consistent Penrose limit, the gauge coupling constant should be scaled to zero, making the limit theory Abelian. Then, contrary to what happens in ungauged supergravity theories, the Penrose limit relates two different gauge supergravity theories.

We have analyzed the properties of the resulting pp-wave solution showing that an enhancement of supersymmetry takes place as it usually occurs when a supergravity solution is transformed to a plane wave geometry. We have also discussed the uplifting of the pp-wave configuration so that it becomes a solution of $N=1$ supergravity in $d=10$ dimensions. In this respect, let us recall that, apart from its intrinsic interest, the $\mathrm{CV}$ solution proved to be very useful in finding a smooth solution of the sevendimensional supergravity model that was analyzed by Maldacena and Núñez in their study of the large $N$ limit of pure $\mathcal{N}=1$ super-Yang-Mills theory [18]. The corresponding Penrose limit was studied in [19] where it was shown that also a supersymmetry enhancement takes place as we have shown it happens for the original CV solution. It would be interesting to explore this enhancement in the uplifted solution as well as its consequences for the related gauge theories. We hope to analyze this issues in a forthcoming work.

\section{Acknowledgements}

We thank Carlos Núñez, Peter Forgacs, Martín Schvellinger and Mikhail Volkov for very useful com- ments and insights. S.R. acknowledges the Universidad de La Plata for hospitality. This work was partially supported by UNLP, CICBA, CONICET, ANPCYT (PICT grant 03-05179) Argentina and ECOS-Sud Argentina-France Collaboration (grant A01E02). E.F.M. is partially supported by Fundación Antorchas, Argentina.

\section{References}

[1] M. Blau, J. Figueroa-O'Farrill, C. Hull, G. Papadopoulos, JHEP 0201 (2002) 047.

[2] R.R. Metsaev, Nucl. Phys. B 625 (2002) 70.

[3] M. Blau, J. Figueroa-O'Farrill, C. Hull, G. Papadopoulos, Class. Quantum Grav. 19 (2002) L87.

[4] D. Berenstein, J.M. Maldacena, H. Nastase, JHEP 0204 (2002) 013.

[5] R.R. Metsaev, A.A. Tseytlin, Phys. Rev. D 65 (2002) 126004.

[6] M. Blau, J. Figueroa-O'Farrill, G. Papadopoulos, Class. Quantum Grav. 19 (2002) 4753.

[7] L.A. Pando Zayas, J. Sonnenschein, JHEP 0205 (2002) 010.

[8] D. Berenstein, E. Gava, J.M. Maldacena, K.S. Narain, H. Nastase, hep-th/0203249.

[9] M. Cvetič, H. Lu, C.N. Pope, Nucl. Phys. B 644 (2002) 65.

[10] R. Penrose, in: M. Cahen, M. Flato (Eds.), Differential Geometry and Relativity, Reidel, Dordrecht, 1976.

[11] R. Güven, Phys. Lett. B 482 (2000) 255.

[12] D.Z. Freedman, J.H. Schwarz, Nucl. Phys. B 137 (1978) 333.

[13] A.H. Chamseddine, M.S. Volkov, Phys. Rev. Lett. 79 (1997) 3343.

[14] A.H. Chamseddine, M.S. Volkov, Phys. Rev. D 57 (1998) 6242 .

[15] E.B. Bogomol'nyi, Sov. J. Nucl. Phys. 24 (1976) 449, Yad. Fiz. 24 (1976) 861.

[16] M.K. Prasad, C.M. Sommerfield, Phys. Rev. Lett. 35 (1975) 760.

[17] C.N. Yang, T.T. Wu, in: H. March, S. Fernbach (Eds.), Properties of Matter Under Unusual Conditions, Interscience, New York, 1969, pp. 349-354.

[18] J.M. Maldacena, C. Núñez, Phys. Rev. Lett. 86 (2001) 588.

[19] J. Gomis, H. Ooguri, Nucl. Phys. B 635 (2002) 106. 